MS11-03

\section{Grouper Iridovirus GIV66: a Bcl-2 protein that inhibits apoptosis by exclusively sequestering Bim}

Sofia Caria ${ }^{1}$,Suresh Banjara ${ }^{2}$, Timothy M. Ryan ${ }^{3}$, Marc Kvansakul ${ }^{2}$

1. La Trobe Institute for Molecular Science, La Trobe University and Australian Synchrotron, ANSTO, Bundoora \& Clayton, Australia

2. La Trobe Institute for Molecular Science, La Trobe University, Bundoora, Australia

3. Australian Synchrotron, ANSTO, Clayton, Australia

email: sofia.caria@synchrotron.org.au

Programmed cell death or apoptosis is a critical mechanism for the controlled removal of damaged or infected cells, and proteins of the Bcl-2 family are important arbiters of this process. Viruses encode functional and structural homologs of Bcl-2 to highjack host cell apoptosis and ensure viral proliferation and/or survival. Grouper iridovirus (GIV) is a large DNA virus belonging to the iridoviridae family that harbors GIV66, a putative Bcl-2 like protein. GIV66 is a mitochondrially localized inhibitor of apoptosis, however the molecular and structural basis of apoptosis inhibition is currently not understood. Our studies show that GIV66 harbors an unusually high level of specificity for pro-apoptotic Bcl-2, and only engages with Bim. We then determined crystal structures of both apoGIV66 as well as bound to Bim BH3. Unexpectedly, apoGIV66 forms dimers via a novel interface that occludes access to the canonical Bcl-2 ligand binding groove, which break apart upon Bim binding. SAXS studies were crucial to further eluciate the mechanism of action of GIV66 in inhibiting apoptosis. GIV66 dimerization seems to impacts on the ability of GIV66 to bind and select host pro-death Bcl-2 protein.
MS11-04

\section{Crystal structures of bacteriophage receptor binding proteins}

Mark Johan van Raaij ${ }^{1}$, Marta Sanz-Gaitero², Antonio PichelBeleiro $^{2}$, Mateo Seoane-Blanco ${ }^{2}$

1. Departamento de Estructura de Macromoleculas, Centro Nacional de Biotecnologia, CSIC, Madrid, Spain

2. ñ CNB-CSIC, Madrid, Spain

email: mjvanraaij@cnb.csic.es

Bacteriophages have specialized receptor binding proteins for initial, reversible, host cell wall recognition. Once a suitable host is found, the phage commits to infection by irreversible attachment via a secondary receptor interaction. The crystal structures of several of these receptor-binding proteins have been solved and have been shown to be mainly beta-structured, but structurally highly diverse and containing several new protein folds. Structures of the receptor-binding proteins of the coliphages T4, T5 and T7, of the Salmonella phage epsilon 15 and of the Staphylococcus phages S241 and K will be shown.

Ongoing structural, mutational and binding analysis of virus receptor-binding proteins with receptors and receptor analogues will be discussed. Bacteriophage receptor-recognizing proteins may be used for bacterial detection, while modification by natural or experimental mutation of bacteriophage receptorbinding domains may allow retargeting of phages to alternative host bacteria. Their shape and stability may also allow their use in nanotechnological applications.

Keywords: Antibiotic resistance, bacterial detection, proteinligand interaction

Keywords: viral Bcl-2, SAXS, crystallography, 